\title{
Synthesis and Anti Bacterial and Anti-ulcer Evaluation of New S-mannich Bases of 4,6-diaryl-3,4-dihydropyrimidin-2(1H)-thiones
}

\author{
Venkateshwarlu Kodhati ${ }^{\dagger}$, Malla Reddy Vanga, and Narsimha Reddy Yellu ${ }^{\dagger, *}$ \\ Divis research centre, Divis Laboratories Ltd., B-34, Industrial Estate, Sanath Nagar, Hyderabad - 500018, India. \\ ${ }^{*}$ E-mail:ynrku@yahoo.co.in \\ †University College of Pharmaceutical Sciences, Kakatiya University, Warangal - 506009, India
}

(Received December 26, 2012; Accepted March 5, 2013)

\begin{abstract}
The synthesis of title compounds were accomplished by synthetic sequence shown in Scheme 1. Chalcones on cyclocondensation with thiourea in ethanol and potassium hydroxide under reflux yielded the respective dihydropyramidin2(1H)-thiones. Each of the dihydropyrimidin thiones was, then subjected to the Mannich condensation in alkaline medium using three different secondary amines, viz., dimethylamine, diethylamine and morpholine to obtain a new series of S-Mannich bases. All the synthesised compounds $\left(\mathbf{C}_{1}-\mathbf{C}_{15}\right)$ were evaluated for their antiulcer and antibacterial activities. Compounds $\mathbf{C}_{4}, \mathbf{C}_{5}, \mathbf{C}_{6}, \mathbf{C}_{14}$ and $\mathbf{C}_{15}$ exhibited relatively more potent antiulcer activity but not comparable to the standard; Omeprazole, while $\mathbf{C}_{\mathbf{1}}, \mathbf{C}_{\mathbf{2}}, \mathbf{C}_{\mathbf{3}}$ and $\mathbf{C}_{\mathbf{1 3}}$ were moderate in activity at $100 \mathrm{mg} / \mathrm{kg}$ p.o. All the compounds $\left(\mathbf{C}_{\mathbf{1}}-\mathbf{C}_{\mathbf{1 5}}\right)$ showed mild to moderate activity against both Gram-positive (S.aureus, L.delbrueckii) and Gram-negative (P.vulgaris, E.coli) bacteria. Amongst the compounds tested, only $\mathbf{C}_{6}, \mathbf{C}_{9}, \mathbf{C}_{12}$ and $\mathbf{C}_{15}$ were found to be potent.
\end{abstract}

Key words: 4, 6-diaryl-3,4-dihydropyramidin-2(1H)-thiones, Anti-ulcer activity, Antibacterial activity

\section{INTRODUCTION}

Pyrimidines are of considerable chemical and biological significance. Many pyrimidine derivatives have displayed diverse pharmacological activities such as anti-inflammatory, ${ }^{1-3}$ antimicrobial, ${ }^{4}$ analgesic, ${ }^{5}$ antiplatelet, ${ }^{6}$ antithrombotic, ${ }^{6}$ antineoplastic, ${ }^{7}$ antianginal, ${ }^{8}$ antiulcer ${ }^{9}$ activities, etc., keeping in view the importance of these dihydropyrimidines in the field of medicine and biology and in continuation of our work on new dihydropyrimidine derivatives, an attempt has now been made to synthesize novel SMannich bases and to investigate their antiulcer and antibacterial activities.

The present work describes the synthesis of S-Mannich bases of dihydropyrimidin-2(1H)-thiones and evalution of antiulcer activity using aspirin-induced model and antibacterial activity against Gram- $(+\mathrm{ve})$ (S.aureus, L.delbrueckii) and Gram-(-ve) (P.vulgaris, E.coli) bacteria. A new series of S-Mannich bases of dihydropyrimidin-2(1H)thiones $\left(\mathbf{C}_{1}-\mathbf{C}_{15}\right)$ were synthesized by the Mannich reaction on five different 3,4-dihyropyrimidine $2(1 H)$-thiones (B1-B5) using aqueous formaldehyde, potassium carbonate and three different secondary amines, viz., dimetylamine, diethylamine and morpholine. All the compounds prepared were characterized by their physical and spectral (IR, NMR, Mass) data. Results of biological activities showed that some of these new S-Mannich bases possess antiulcer and antibacterial activities, with a variation in potencies.

\section{RESULTS AND DISCUSSION}

\section{Chemistry}

A novel series of [4,6-substituted diaryl-3,4-dihyropyramidine-2-yl-thiomethyl]-amines were synthesized on the lines of a synthetic route presented in Scheme 1. The desired compounds were synthesized as follows: initially, an equimolar quantities of differently substituted acetophenones and substituted benzaldehydes in the presence of sodium hydroxide, through a mixed aldol condensation yielded the corresponding chalcones $\left(\mathbf{A}_{\mathbf{1}}-\mathbf{A}_{\mathbf{5}}\right)$. Each of these chalcones $\left(\mathbf{A}_{\mathbf{1}}-\mathbf{A}_{\mathbf{5}}\right)$ was heated under reflux with thiourea in the presence of sodium hydroxide to yield the respective 4,6-diaryl dihydropyrimidine-2(1H)-thione $\left(\mathbf{B}_{1}-\right.$ $\left.\mathbf{B}_{5}\right)$. The Mannich condensation of thiones, $\mathbf{B}_{1}-\mathbf{B}_{5}$ with three secondary amines (dimethylamine, diethylamine and morpholine) and formaldehyde, in dimethyl sulfoxide (DMSO) afforded the corresponding Mannich bases $\left(\mathbf{C}_{\mathbf{1}}-\mathbf{C}_{\mathbf{1 5}}\right)$. These compounds have been characterized by their IR, ${ }^{1}$ HNMR and Mass spectral data and elemental analyses. The physical data of the chalcones and dihydropyrimidin2(1H)-thiones are presented in Table 1 and that of their S- 


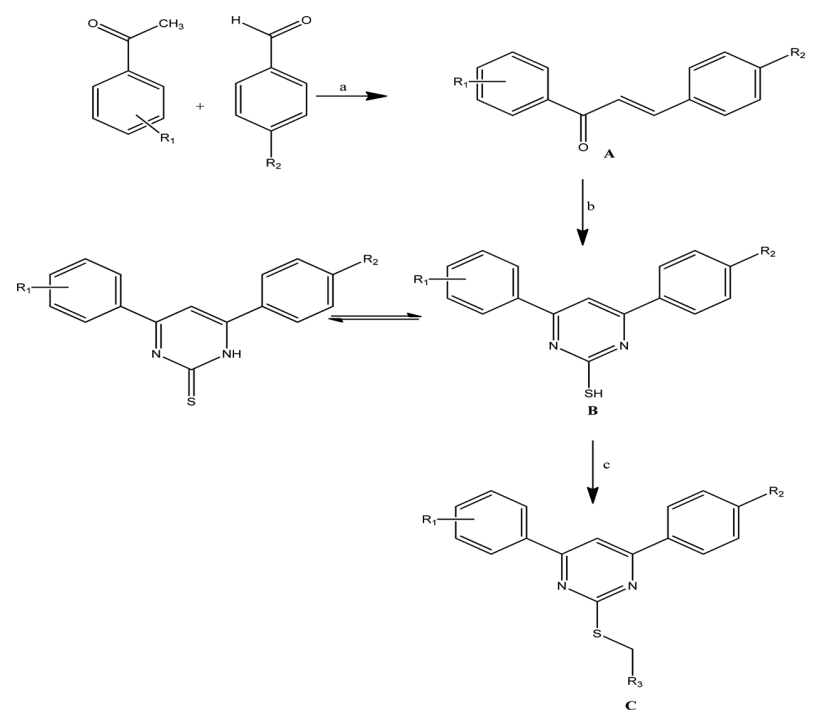

Scheme 1. Substituents: $\mathrm{R}_{1}=p-\mathrm{Cl}, p-\mathrm{OH}, m-\mathrm{NO}_{2} ; \mathrm{R}_{2}=p-\mathrm{Cl}$, $p-\mathrm{OCH}_{3}, p-\mathrm{N}\left(\mathrm{CH}_{3}\right)_{2} ; \mathrm{R}_{3}=$ dimethylamino, diethylamino \& 4morpholino. Reaction conditions: $\mathrm{a}=\mathrm{aq} . \mathrm{NaOH}$, ethanol; $\mathrm{b}=$ thiourea, $\mathrm{KOH}$, ethanol; $\mathrm{c}=$ secondary amines (dimethylamine, diethylamine or morpholine), aq.formaldehyde (37\%), $\mathrm{K}_{2} \mathrm{CO}_{3}$ and DMSO.

Mannich bases in Table 2, respectively.

\section{Spectral Analyses}

A sharp singlet peak at $\delta 3.40 \mathrm{ppm}$ indicates the presence of $\mathbf{C H}$ proton ( $\mathrm{C}_{5}$ of pyrimidine), a characteristic signal at $\delta 3.62 \mathrm{ppm}$ as a singlet is assigned to $\mathbf{C H}_{2}$ proton (S$\mathbf{C H}_{2}-\mathbf{N}$ ), a multiplet at $\delta 7.2-7.8 \mathrm{ppm}$ indicates the presence of aromatic protons $(\mathrm{Ar}-\mathrm{H})$ The mass spectra of all the synthesized compounds $\left(\mathbf{C}_{1}-\mathbf{C}_{15}\right)$ showed molecular ion peak at $\mathrm{M}+1$ and $\mathrm{M}+2$, characteristic of the presence of sulphur.

\section{BIOLOGICAL EVALUATION}

\section{Antiulcer Activity ${ }^{13-16}$}

Antiulcer activity of all the synthesized compounds was evaluated by using aspirin induced ulcer model in Albino rats. Albino rats weighing $150-200 \mathrm{~g}$ were divided into different groups consisting of six animals per group and starved for $24 \mathrm{hrs}$. The test compounds were administered orally $30 \mathrm{~min}$ prior to aspirin administration $(100 \mathrm{mg} / \mathrm{kg})$. Control group received only vehicle ( $1 \%$ sodium carboxy methyl cellulose), whereas the standard group received omeprazole $20 \mathrm{mg} / \mathrm{kg}$. After $5 \mathrm{hr}$ of drug treatment all the animals were sacrificed according to the CPCSEA guidelines and stomach was cut open in the greater curvature, washed with ice cold saline and cleaned. The gastric mucosa was examined for ulcer scoring by using $4 \mathrm{x}$ binocular magnifier . The ulcer score is according to its severity in comparison with that of the standard. Ulcer scores were recorded as follows:
0 : Normal, no ulcer
1: Isolated haemorrhagic spot
2: Dense haemorrhagic spot
3: Small ulcer
4: Large ulcer
5: Perforation

The severity of the mucosal damage was assessed by Ulcer Index (UI) and it was calculated by using the formula:

$$
\begin{aligned}
& \mathrm{UI}=\mathrm{U}_{\mathrm{N}}+\mathrm{U}_{\mathrm{S}}+\mathrm{U}_{\mathrm{P}} \times 10^{-1} \\
& \mathrm{U}_{\mathrm{N}}=\text { average of number of ulcers per animal, } \\
& \mathrm{U}_{\mathrm{S}}=\text { average of severity score, and } \\
& \mathrm{U}_{\mathrm{P}}=\text { percentage of animals with ulcers. }
\end{aligned}
$$

\begin{tabular}{|c|c|c|c|c|c|c|c|}
\hline Code & $\mathrm{R}_{1}$ & $\mathrm{R}_{2}$ & Mol. formula & Mol. Weight & M.P & $\%$ Yield & $\mathrm{R}_{\mathrm{f}}^{*}$ \\
\hline $\mathbf{A}_{1}$ & $\mathrm{p}-\mathrm{Cl}$ & $\mathrm{p}-\mathrm{Cl}$ & $\mathrm{C}_{15} \mathrm{H}_{10} \mathrm{Cl}_{2} \mathrm{O}$ & 276 & $95-100$ & 67 & 0.71 \\
\hline $\mathbf{A}_{2}$ & $\mathrm{p}-\mathrm{Cl}$ & $\mathrm{p}-\mathrm{OCH}_{3}$ & $\mathrm{C}_{15} \mathrm{H}_{13} \mathrm{ClO}_{2}$ & 260 & $79-82$ & 62 & 0.68 \\
\hline $\mathbf{A}_{3}$ & $\mathrm{p}-\mathrm{Cl}$ & $\mathrm{p}-\mathrm{N}\left(\mathrm{CH}_{3}\right)_{2}$ & $\mathrm{C}_{17} \mathrm{H}_{16} \mathrm{ClNO}$ & 285 & $87-91$ & 67 & 0.67 \\
\hline $\mathbf{A}_{4}$ & $\mathrm{p}-\mathrm{OH}$ & $\mathrm{p}-\mathrm{Cl}$ & $\mathrm{C}_{15} \mathrm{H}_{11} \mathrm{ClO}_{2}$ & 258 & $85-87$ & 69 & 0.54 \\
\hline$A_{5}$ & $\mathrm{~m}-\mathrm{NO}_{2}$ & $\mathrm{p}-\mathrm{Cl}$ & $\mathrm{C}_{15} \mathrm{H}_{10} \mathrm{ClNO}_{3}$ & 287 & $92-96$ & 65 & 0.63 \\
\hline $\mathbf{B}_{1}$ & $\mathrm{P}-\mathrm{Cl}$ & $\mathrm{p}-\mathrm{Cl}$ & $\mathrm{C}_{16} \mathrm{H}_{11} \mathrm{Cl}_{2} \mathrm{~N}_{2} \mathrm{O}$ & 317 & $155-160$ & 59 & 0.49 \\
\hline $\mathbf{B}_{2}$ & $\mathrm{P}-\mathrm{Cl}$ & $\mathrm{p}-\mathrm{OCH}_{3}$ & $\mathrm{C}_{16} \mathrm{H}_{14} \mathrm{ClN}_{2} \mathrm{O}_{2}$ & 301 & $174-178$ & 49 & 0.52 \\
\hline $\mathbf{B}_{3}$ & $\mathrm{P}-\mathrm{Cl}$ & $\mathrm{p}-\mathrm{N}\left(\mathrm{CH}_{3}\right)_{2}$ & $\mathrm{C}_{18} \mathrm{H}_{17} \mathrm{ClN}_{3} \mathrm{O}$ & 326 & $163-165$ & 45 & 0.47 \\
\hline $\mathbf{B}_{4}$ & $\mathrm{P}-\mathrm{OH}$ & $\mathrm{p}-\mathrm{Cl}$ & $\mathrm{C}_{16} \mathrm{H}_{13} \mathrm{ClN}_{2} \mathrm{O}_{2}$ & 300 & $168-170$ & 43 & 0.55 \\
\hline $\mathbf{B}_{5}$ & $\mathrm{~m}-\mathrm{NO}_{2}$ & $\mathrm{p}-\mathrm{Cl}$ & $\mathrm{C}_{16} \mathrm{H}_{11} \mathrm{ClN}_{3} \mathrm{O}_{3}$ & 329 & $182-185$ & 59 & 0.47 \\
\hline
\end{tabular}

The mean ulcer index and percentage protection produced

Table 1. Physical data of chalcones and dihydropyrimidin-2(1H)-thiones

*n-hexane-ethyl acetate (1:1) on silica gel. 
Table 2. Physical data of S-Mannich bases of dihydropyrimidin-2(1H)-thiones

\begin{tabular}{|c|c|c|c|c|c|c|c|c|}
\hline Code & $\mathrm{R}_{1}$ & $\mathrm{R}_{2}$ & $\mathrm{R}_{3}$ & Mol. formula & Mol. weight & M.P $\left({ }^{\circ} \mathrm{C}\right)$ & $\%$ Yield & $\mathrm{R}_{\mathrm{f}}^{*}$ \\
\hline $\mathrm{C}_{1}$ & $\mathrm{p}-\mathrm{Cl}$ & $\mathrm{p}-\mathrm{Cl}$ & dimethylamino & $\mathrm{C}_{17} \mathrm{H}_{14} \mathrm{Cl}_{2} \mathrm{~N}_{3} \mathrm{~S}$ & 390 & $268-270$ & 56 & 0.50 \\
\hline $\mathbf{C}_{2}$ & $\mathrm{p}-\mathrm{Cl}$ & $\mathrm{p}-\mathrm{Cl}$ & diethylamino & $\mathrm{CH}_{21} \mathrm{Cl}_{2} \mathrm{~N}_{3} \mathrm{~S}$ & 417 & $248-252$ & 60 & 0.44 \\
\hline $\mathrm{C}_{3}$ & $\mathrm{p}-\mathrm{Cl}$ & $\mathrm{p}-\mathrm{Cl}$ & 4-morpholino & $\mathrm{CH}_{19} \mathrm{Cl}_{2} \mathrm{~N}_{3} \mathrm{OS}$ & 431 & $264-268$ & 64 & 0.48 \\
\hline $\mathrm{C}_{4}$ & $\mathrm{p}-\mathrm{Cl}$ & $\mathrm{p}-\mathrm{OCH}_{3}$ & dimethylamino & $\mathrm{C}_{20} \mathrm{H}_{20} \mathrm{ClN}_{3} \mathrm{OS}$ & 385 & $254-256$ & 56 & 0.42 \\
\hline $\mathrm{C}_{5}$ & $\mathrm{p}-\mathrm{Cl}$ & $\mathrm{p}-\mathrm{OCH}_{3}$ & diethylamino & $\mathrm{C}_{24} \mathrm{H}_{22} \mathrm{ClN}_{3} \mathrm{OS}$ & 413 & $264-266$ & 60 & 0.40 \\
\hline $\mathrm{C}_{6}$ & $\mathrm{p}-\mathrm{Cl}$ & $\mathrm{p}-\mathrm{OCH}_{3}$ & 4-morpholino & $\mathrm{C}_{22} \mathrm{H}_{22} \mathrm{ClN}_{3} \mathrm{O}_{2} \mathrm{~S}$ & 427 & $250-254$ & 54 & 0.50 \\
\hline $\mathrm{C}_{7}$ & $\mathrm{p}-\mathrm{Cl}$ & $\mathrm{p}-\mathrm{N}\left(\mathrm{CH}_{3}\right)_{2}$ & dimethylamino & $\mathrm{C}_{21} \mathrm{H}_{23} \mathrm{ClN}_{4} \mathrm{~S}$ & 398 & $268-270$ & 56 & 0.51 \\
\hline $\mathrm{C}_{8}$ & $\mathrm{p}-\mathrm{Cl}$ & $\mathrm{p}-\mathrm{N}\left(\mathrm{CH}_{3}\right)_{2}$ & diethylamino & $\mathrm{C}_{23} \mathrm{H}_{27} \mathrm{ClN}_{4} \mathrm{~S}$ & 426 & $276-278$ & 60 & 0.57 \\
\hline $\mathrm{C}_{9}$ & $\mathrm{p}-\mathrm{Cl}$ & $\mathrm{p}-\mathrm{N}\left(\mathrm{CH}_{3}\right)_{2}$ & 4-morpholine & $\mathrm{C}_{23} \mathrm{H}_{25} \mathrm{ClN}_{4} \mathrm{OS}$ & 440 & $284-286$ & 64 & 0.59 \\
\hline $\mathrm{C}_{10}$ & $\mathrm{p}-\mathrm{OH}$ & $\mathrm{p}-\mathrm{Cl}$ & dimethylamino & $\mathrm{C}_{19} \mathrm{H}_{18} \mathrm{ClN}_{3} \mathrm{OS}$ & 310 & $279-282$ & 56 & 0.50 \\
\hline $\mathrm{C}_{11}$ & $\mathrm{p}-\mathrm{OH}$ & $\mathrm{p}-\mathrm{Cl}$ & diethylamino & $\mathrm{C}_{21} \mathrm{H}_{22} \mathrm{ClN}_{3} \mathrm{OS}$ & 399 & $285-288$ & 60 & 0.54 \\
\hline $\mathrm{C}_{12}$ & $\mathrm{p}-\mathrm{OH}$ & $\mathrm{p}-\mathrm{Cl}$ & 4-morpholino & $\mathrm{C}_{21} \mathrm{H}_{20} \mathrm{ClN}_{3} \mathrm{O}_{2} \mathrm{~S}$ & 413 & $274-276$ & 64 & 0.58 \\
\hline $\mathrm{C}_{13}$ & $\mathrm{~m}-\mathrm{NO}_{2}$ & $\mathrm{p}-\mathrm{Cl}$ & dimethylamino & $\mathrm{C}_{19} \mathrm{H}_{17} \mathrm{ClN}_{4} \mathrm{O}_{2} \mathrm{~S}$ & 400 & $268-270$ & 56 & 0.47 \\
\hline $\mathrm{C}_{14}$ & $\mathrm{~m}-\mathrm{NO}_{2}$ & $\mathrm{p}-\mathrm{Cl}$ & diethylamino & $\mathrm{C}_{21} \mathrm{H}_{19} \mathrm{ClN}_{4} \mathrm{O}_{3} \mathrm{~S}$ & 428 & $275-278$ & 60 & 0.50 \\
\hline $\mathrm{C}_{15}$ & $\mathrm{~m}-\mathrm{NO}_{2}$ & $\mathrm{p}-\mathrm{Cl}$ & 4-morpholino & $\mathrm{C}_{21} \mathrm{H}_{19} \mathrm{ClN}_{4} \mathrm{O}_{3} \mathrm{~S}$ & 442 & $281-284$ & 71 & 0.48 \\
\hline
\end{tabular}

*n-hexane-ethyl acetate (1:2) on silica gel.

Table 3. Results of antiulcer activity of different compounds

\begin{tabular}{|c|c|c|c|c|c|}
\hline Code & $\mathrm{R}_{1}$ & $\mathrm{R}_{2}$ & $\mathrm{R}_{3}$ & Ulcer index (UI)* & $\%$ protection \\
\hline $\mathrm{C}_{1}$ & $\mathrm{p}-\mathrm{Cl}$ & $\mathrm{p}-\mathrm{Cl}$ & dimethylamino & $19.23 \pm 4.2^{*}$ & 30.73 \\
\hline $\mathrm{C}_{2}$ & $\mathrm{p}-\mathrm{Cl}$ & $\mathrm{p}-\mathrm{Cl}$ & diethylamino & $19.56 \pm 3.8^{*}$ & 29.54 \\
\hline $\mathbf{C}_{3}$ & $\mathrm{p}-\mathrm{Cl}$ & $\mathrm{p}-\mathrm{Cl}$ & 4-morpholino & $17.96 \pm 4.2^{* *}$ & 35.30 \\
\hline $\mathrm{C}_{4}$ & $\mathrm{p}-\mathrm{Cl}$ & $\mathrm{p}-\mathrm{OCH}_{3}$ & dimethylamino & $12.50 \pm 0.52^{* * *}$ & 54.97 \\
\hline $\mathrm{C}_{5}$ & $\mathrm{p}-\mathrm{Cl}$ & $\mathrm{p}-\mathrm{OCH}_{3}$ & diethylamino & $11.66 \pm 0.23^{* * *}$ & 58.00 \\
\hline $\mathrm{C}_{6}$ & $\mathrm{p}-\mathrm{Cl}$ & $\mathrm{p}-\mathrm{OCH}_{3}$ & 4-morpholino & $11.33 \pm 0.09^{* * *}$ & 59.19 \\
\hline $\mathbf{C}_{7}$ & $\mathrm{p}-\mathrm{Cl}$ & $\mathrm{p}-\mathrm{N}\left(\mathrm{CH}_{3}\right)_{2}$ & dimethylamino & $22.66 \pm 5.3^{\text {ns }}$ & 18.37 \\
\hline $\mathrm{C}_{8}$ & $\mathrm{p}-\mathrm{Cl}$ & $\mathrm{p}-\mathrm{N}\left(\mathrm{CH}_{3}\right)_{2}$ & diethylamino & $23.58 \pm 6.1^{\mathrm{ns}}$ & 15.06 \\
\hline $\mathrm{C}_{9}$ & $\mathrm{p}-\mathrm{Cl}$ & $\mathrm{p}-\mathrm{N}\left(\mathrm{CH}_{3}\right)_{2}$ & 4-morpholine & $18.16 \pm 4.2^{* *}$ & 34.58 \\
\hline $\mathrm{C}_{10}$ & $\mathrm{p}-\mathrm{OH}$ & $\mathrm{p}-\mathrm{Cl}$ & dimethylamino & $27.36 \pm 6.2^{\text {ns }}$ & 1.44 \\
\hline $\mathrm{C}_{11}$ & $\mathrm{p}-\mathrm{OH}$ & $\mathrm{p}-\mathrm{Cl}$ & diethylamino & $27.00 \pm 6.4^{\mathrm{ns}}$ & 2.74 \\
\hline $\mathrm{C}_{12}$ & $\mathrm{p}-\mathrm{OH}$ & $\mathrm{p}-\mathrm{Cl}$ & 4-morpholino & $26.50 \pm 7.8^{\text {ns }}$ & 4.54 \\
\hline $\mathrm{C}_{13}$ & $\mathrm{~m}-\mathrm{NO}_{2}$ & $\mathrm{p}-\mathrm{Cl}$ & dimethylamino & $17.98 \pm 5.3^{* *}$ & 35.23 \\
\hline $\mathrm{C}_{14}$ & $\mathrm{~m}-\mathrm{NO}_{2}$ & $\mathrm{p}-\mathrm{Cl}$ & diethylamino & $12.50 \pm 0.98^{* * *}$ & 54.97 \\
\hline $\mathrm{C}_{15}$ & $\mathrm{~m}-\mathrm{NO}_{2}$ & $\mathrm{p}-\mathrm{Cl}$ & 4-morpholino & $12.80 \pm 0.68^{* * *}$ & 53.89 \\
\hline Control & - & - & - & $27.76 \pm 7.3$ & - \\
\hline Standard & - & - & - & $0.25 \pm 0.06^{* * *}$ & 99.10 \\
\hline
\end{tabular}

*Ulcer index expressed in Mean \pm S.D.

Test dose for control, standard and test compounds: $100 \mathrm{mg} / \mathrm{kg}$.

$\mathrm{n}=6$, values expressed as Mean $\pm \mathrm{SD}$, statistical comparison was performed using One-Way ANOVA followed by Dunnett's post-test, ${ }^{*} p \leq$ $0.05,{ }^{* *} p \leq 0.01,{ }^{* * *} p \leq 0.001,{ }^{\text {ns }}-$ non significant, compared to control.

by different test compounds are presented in Table 3 along with their statistical significance.

In vivo antiulcer activity of the synthesized compounds $\left(\mathbf{C}_{\mathbf{1}}-\mathbf{C}_{\mathbf{1 5}}\right)$ was evaluated by aspirin-induced ulcer model using Omeprazole as the standard drug. Ulcer index and percentage protection of test compounds was calculated. From the results, it could be inferred that the compunds
$\mathbf{C}_{4}, \mathbf{C}_{5}, \mathbf{C}_{6}, \mathbf{C}_{14}$ and $\mathbf{C}_{15}$ possess more antiulcer activity while compounds $\mathbf{C}_{\mathbf{1}}, \mathbf{C}_{\mathbf{2}}, \mathbf{C}_{\mathbf{3}}, \mathbf{C}_{\mathbf{9}}$ and $\mathbf{C}_{\mathbf{1 3}}$ were moderate in their activity when compared with the control group. The compounds $\mathbf{C}_{\mathbf{7}}, \mathbf{C}_{\mathbf{8}}, \mathbf{C}_{\mathbf{1 0}}, \mathbf{C}_{\mathbf{1 1}}$ and $\mathbf{C}_{\mathbf{1 2}}$ were lacking significant activity. However, the antiulcer activity of the present series of compounds is not at all comparable with that of the standard Omeprazole. 
Table 4. Antibacterial activity of synthesised compounds $\left(\mathbf{C}_{1}-\mathbf{C}_{15}\right)$ and their MIC $(\mu \mathrm{g} / \mathrm{ml})$

\begin{tabular}{ccccc}
\hline Code & S.aureus & L.delbrueckii & P.vulgaris & E.coli \\
\hline Ciprofloxacin & 10 & 10 & 10 & 10 \\
$\mathbf{C}_{\mathbf{1}}$ & 50 & 25 & 50 & 75 \\
$\mathbf{C}_{\mathbf{2}}$ & 60 & 75 & 60 & 100 \\
$\mathbf{C}_{\mathbf{3}}$ & 50 & 25 & 60 & 50 \\
$\mathbf{C}_{\mathbf{4}}$ & 75 & 100 & 100 & 50 \\
$\mathbf{C}_{\mathbf{5}}$ & 100 & 100 & 50 & 50 \\
$\mathbf{C}_{\mathbf{6}}$ & 30 & 25 & 25 & 35 \\
$\mathbf{C}_{\mathbf{7}}$ & 25 & 50 & 100 & 25 \\
$\mathbf{C}_{\mathbf{8}}$ & 100 & 100 & 25 & 25 \\
$\mathbf{C}_{\mathbf{9}}$ & 25 & 55 & 45 & 35 \\
$\mathbf{C}_{\mathbf{1 0}}$ & 50 & 125 & 50 & 25 \\
$\mathbf{C}_{\mathbf{1 1}}$ & 25 & 50 & 100 & 125 \\
$\mathbf{C}_{\mathbf{1 2}}$ & 50 & 35 & 60 & 75 \\
$\mathbf{C}_{\mathbf{1 3}}$ & 125 & 25 & 50 & 50 \\
$\mathbf{C}_{\mathbf{1 4}}$ & 100 & 100 & 125 & 50 \\
$\mathbf{C}_{\mathbf{1 5}}$ & 25 & 50 & 35 & 25 \\
\hline
\end{tabular}

\section{Antibacterial Activity ${ }^{17}$}

The antibacterial activity of the test compounds was assayed, against four different strains of bacteria, i.e., Staphylococcus aureus and Lactobacillus delbrueckii (Gram positive) Proteus vulgaris and Escherichia coli (Gram negative) by the agar diffusion method. Generally, the antibacterial activity of a compound is expressed in terms of its ability to inhibit the growth of bacteria in nutrient broth or agar. Ciproflaxacin was used as the standard drug. The compounds and their minimum inhibitory concentrations (MIC) in $\mu \mathrm{g} / \mathrm{ml}$ are presented in Table 4.

In vitro antibacterial activity of all the synthesized compounds $\left(\mathbf{C}_{1}-\mathbf{C}_{15}\right)$ showed to be mild to moderate against both the Gram-positive (S.aureus, L.delbrueckii) and Gramnegative (P.vulgaris, E.coli) bacteria. Amongst them, the compounds $\mathbf{C}_{6}, \mathbf{C}_{9}, \mathbf{C}_{12}$ and $\mathbf{C}_{15}$ were found to be relatively more potent.

\section{EXPERIMENTAL SECTION}

All the chemicals were of synthetic grade and commercially procured from Sigma Aldrich, Mumbai, India. Melting points were determined by open capillary method and were uncorrected. IR spectra were recorded on FTIR (Bruker Alpha-E) by $\mathrm{KBr}$ disc method. The ${ }^{1} \mathrm{H}$ NMR spectra were recorded at $400 \mathrm{MHz}$ in DMSO- $\mathrm{d}_{6}$ as solvent and TMS as an internal standard using BRUKER ADVANCE 400 instrument. Mass spectra were recorded on PEP-SCIUX-APIQ pulsar mass spectrophotometer. Elemental analyses were performed on Perkin-Elmer EAL240 elemental analyzer.

\section{General Procedure for Synthesis of Chalcones (A)}

A mixture of $22 \mathrm{gm}$ of sodium hydroxide in $200 \mathrm{ml}$ of water and $100 \mathrm{gm}$ of rectified spirit in a $500 \mathrm{ml}$ bolt head flask was provided with a mechanical stirrer. The flask was immersed in a bath of crushed ice; $52 \mathrm{gm}(0.43 \mathrm{~mol})$ of freshly distilled acetophenone was added while stirring and then followed by $46 \mathrm{gm}$ ( $44 \mathrm{ml}, 0.43 \mathrm{~mol}$ ) of pure benzaldehyde. The temperature of the reaction mixture was maintained at about $25^{\circ} \mathrm{C}$ (limits are: $15-30^{\circ} \mathrm{C}$ ) and stirred vigorously until the reaction mass was so thick that stirring was no longer possible (2-3 hr). Stirrer was removed and the reaction mixture was kept in an ice chest or refrigerator, overnight. The product was filtered and washed with cold water until the washings were neutral to litmus followed with $20 \mathrm{ml}$ of ice cold, rectified spirit and dried. It was purified by recrystallization from ethanol to give a pure compound. ${ }^{10}$

\section{General Procedure for Synthesis of 4,6-diaryl-pyrim- idin-2-thiol (B)}

A mixture of 0.01 moles of chalcone, 0.01 moles of thiourea and potassium hydroxide $(1 \mathrm{gm})$ in $20 \mathrm{ml}$ ethanol was heated under reflux for $6 \mathrm{hr}$. The reaction was monitored by TLC. After completion of the reaction, the contents were cooled to room temperature and poured into ice cold water $(50 \mathrm{ml})$ while stirring. The solid thus resulted was filtered, washed with portions of cold water and dried. It was purified by recrystallization from ethanol to give a pure compound. ${ }^{11}$

\section{General Procedure for the Synthesis of S-Mannich Bases of 4,6-diaryl-dihydropyrimidin-2(1H) Thione (C)}

Dihydropyrimidine thione ( 0.005 moles) was dissolved in dimethyl sulfoxide $(25 \mathrm{ml})$ in a conical flask and stirred with $37 \%$ formaldehyde $(0.01$ moles) then added anhydrous potassium carbonate (1.0gm), appropriate secondary amine $(0.005 \mathrm{~mol})$ and continued the stirring magnetically for about $2 \mathrm{hrs}$. The reaction mixture was then heated under reflux for about $5 \mathrm{hrs}$. Completion of reaction was confirmed by TLC and then kept in refrigerator for $48 \mathrm{hr}$, filtered the product, washed with small portions of cold water and dried. The crude product was purified by recrystallization from petroleum ether-chloroform $(1: 1)$ mixture. ${ }^{12}$ 
Characterization Data of Synthesised S-Mannich Bases

$\left(\mathrm{C}_{1}-\mathrm{C}_{15}\right)$

1-((4,6-bis(4-chlorophenyl)pyrimidin-2-yl)thio)-N, Ndimethylmethanamine $\left(C_{1}\right)$ :

IR( $\left.\mathrm{KBr}, \mathrm{cm}^{-1}\right): 3050$ (=C-H str, aromatic), $1648(\mathrm{C}=\mathrm{N}$ str, pyrimidine), 1602 and $1425(\mathrm{C}=\mathrm{C}$ str, aromatic), 770 (C-Cl str), 620 (C-S-C str); ${ }^{1} \mathbf{H}$ NMR (400Hz, DMSO-d 6 ) $\delta$ ppm: 2.26(s, 6H, 2CH $), 4.00$ (s, 2H, $\left.\mathrm{CH}_{2}\right), 7.21$ (s, $1 \mathrm{H}$, CH), 7.28-8.00 (m, 8H, Ar-H); EI-MS: 389; Ana. Calcd. for $\mathrm{C}_{19} \mathrm{H}_{17} \mathrm{Cl}_{2} \mathrm{~N}_{3} \mathrm{~S}$ : C, 58.06; H, 4.39; N, 10.77. Found: C, 58.03; H, 4.35; N, 10.71\%.

\section{$\mathrm{N}$-((4,6-bis(4-chlorophenyl)pyrimidin-yl)thio)methyl)-}

$N$-ethylethanamine $\left(C_{2}\right)$ :

IR(KBr, cm $\left.{ }^{-1}\right): 3048$ (=C-H str, aromatic), 1648(C=N str, pyrimidine) 1605 and $1428(\mathrm{C}=\mathrm{C}$ str, aromatic $), 750$ (C-Cl str), 618 (C-S-C str); ${ }^{1} \mathbf{H}$ NMR (400 Hz, DMSO$\left.\mathrm{d}_{6}\right) \delta$ ppm: $1.04\left(\mathrm{t}, 6 \mathrm{H}, 2 \mathrm{CH}_{3}\right), 2.65\left(\mathrm{q}, 4 \mathrm{H}, 2 \mathrm{CH}_{2}\right), 3.99(\mathrm{~s}$, $\left.2 \mathrm{H}, \mathrm{CH}_{2}\right), 7.20$ (s, 1H, CH) 7.26-8.01 (m, 8H, Ar-H); EIMS: 417; Ana. Calcd. for $\mathrm{C}_{21} \mathrm{H}_{21} \mathrm{Cl}_{2} \mathrm{~N}_{3} \mathrm{~S}$ : C, 60.29; $\mathrm{H}$, 5.06; N, 10.04. Found: C, 60.27; H, 5.02; N, 10.01\%.

\section{4-((4,6-bis(4-chlorophenyl)pyrimidin-2-yl)thio)methyl)mor- pholine $\left(C_{3}\right)$ :}

IR(KBr, cm $\left.{ }^{-1}\right): 3052$ (=C-H str, aromatic), $1645(\mathrm{C}=\mathrm{N}$ str, pyrimidine $) 1601$ and $1426(\mathrm{C}=\mathrm{C}$ str, aromatic $), 755$ (C-Cl str), 619 (C-S-C str); ${ }^{1} \mathbf{H}$ NMR (400 Hz, DMSO$\left.\mathrm{d}_{6}\right) \delta$ ppm: $2.98\left(\mathrm{t}, 4 \mathrm{H}, 2 \mathrm{CH}_{2}\right), 3.68\left(\mathrm{t}, 4 \mathrm{H}, 2 \mathrm{CH}_{2}\right) 3.97(\mathrm{~s}$, $\left.2 \mathrm{H}, \mathrm{CH}_{2}\right), 7.20$ (s, 1H, CH), 7.27-8.00 (m, 8H, Ar-H); EI-MS: 431; Ana. Calcd. for $\mathrm{C}_{21} \mathrm{H}_{19} \mathrm{Cl}_{2} \mathrm{~N}_{3} \mathrm{OS}$ : C, 58.34; H, 4.43; N, 9.72. Found C, 58.36; H, 4.41; N, 9.69\%.

1-((4-(4-chlorophenyl)-6-(4-methoxyphenyl)pyrimidin-2-yl)thio)-N,N-dimthyimethanamine $\left(C_{4}\right)$ :

IR $\left(\mathrm{KBr}, \mathrm{cm}^{-1}\right): 3049(=\mathrm{C}-\mathrm{H}$ str, aromatic $), 1650(\mathrm{C}=\mathrm{N}$ str, pyrimidine) 1602 and $1429(\mathrm{C}=\mathrm{C}$ str, aromatic), 758 (C-Cl str), 622 (C-S-C str); ${ }^{1} \mathbf{H}$ NMR (400 Hz, DMSO$\left.\mathrm{d}_{6}\right) \delta$ ppm: $2.28\left(\mathrm{~s}, 6 \mathrm{H}, 2 \mathrm{CH}_{3}\right), 3.85\left(\mathrm{~s}, 3 \mathrm{H}, \mathrm{CH}_{2}\right), 4.00(\mathrm{~s}$, $\left.2 \mathrm{H}, \mathrm{CH}_{2}\right), 7.22$ (s, 1H, CH), 7.28-8.02 (m, 8H, Ar-H); EI-MS: 385; Ana. Calcd. for $\mathrm{C}_{20} \mathrm{H}_{20} \mathrm{ClN}_{3} \mathrm{OS}$ : C, 62.25; $\mathrm{H}$, 5.22; N, 10.89. Found C, 62.21; H, 5.19; N, 10.85\%.

$\mathrm{N}$-(((4-(4-chlorophenyl)-6-(4-methoxyphenyl)pyrimidin-2-yl)thio)methyl)-N-ethylethanamine $\left(C_{5}\right)$ :

IR $\left(\mathrm{KBr}, \mathrm{cm}^{-1}\right) 3052$ (=C-H str, aromatic), $1645(\mathrm{C}=\mathrm{N}$ str, pyrimidine $) 1605$ and $1430(\mathrm{C}=\mathrm{C}$ str, aromatic $), 760$ (C-Cl str), 625(C-S-C str); ${ }^{1} \mathbf{H}$ NMR (400 Hz, DMSO-d $\left.{ }_{6}\right)$ $\delta$ ppm: $1.02\left(\mathrm{t}, 6 \mathrm{H}, 2 \mathrm{CH}_{3}\right), 2.64\left(\mathrm{q}, 4 \mathrm{H}, 2 \mathrm{CH}_{2}\right), 3.83(\mathrm{~s}, 3 \mathrm{H}$, $\left.\mathrm{OCH}_{3}\right), 3.99\left(\mathrm{~s}, 2 \mathrm{H}, \mathrm{CH}_{2}\right), 7.25(\mathrm{~s}, 1 \mathrm{H}, \mathrm{CH}), 7.29-8.00(\mathrm{~m}, 8 \mathrm{H}$,
Ar-H); EI-MS: 413; Ana. Calcd. for $\mathrm{C}_{24} \mathrm{H}_{22} \mathrm{ClN}_{3} \mathrm{OS}$ : C, 63.83; H, 5.84; N, 10.15. Found C, 63.81; H, 5.80; N, 10.11\%.

4-(((4-(4-chlorophenyl)-6-(4-methoxyphenyl)pyrimidin-2-yl)thio)methyl)morphine $\left(C_{6}\right)$ :

IR $\left(\mathrm{KBr}, \mathrm{cm}^{-1}\right): 3054$ (=C-H str, aromatic), $1651(\mathrm{C}=\mathrm{N}$ str, pyrimidine) 1600 and $1429(\mathrm{C}=\mathrm{C}$ str, aromatic $), 761$ (C-Cl str), 621(C-S-C str); ${ }^{1}$ H NMR (400 Hz, DMSO-d $)$ $\delta$ ppm: $2.96\left(\mathrm{t}, 4 \mathrm{H}, 2 \mathrm{CH}_{2}\right), 3.70\left(\mathrm{t}, 4 \mathrm{H} 2 \mathrm{CH}_{2}\right), 3.84(\mathrm{~s}, 3 \mathrm{H}$, $\left.\mathrm{OCH}_{3}\right), 4.02\left(\mathrm{~s}, 2 \mathrm{H}, \mathrm{CH}_{2}\right), 7.23(\mathrm{~s}, 1 \mathrm{H}, \mathrm{CH}), 7.28-8.02(\mathrm{~m}$, $8 \mathrm{H}, \mathrm{Ar}-\mathrm{H})$; EI-MS: 427; Ana.Calcd.for $\mathrm{C}_{22} \mathrm{H}_{22} \mathrm{ClN}_{3} \mathrm{O}_{2} \mathrm{~S}$ : C, 61.74; H, 5.18; N, 9.82. Found C, 61.71; H, 5.12; N, $9.79 \%$.

4-(6-(4-chlorophenyl)-2-(((dimethylamino)methyl)thio)pyrimidin-4-yl)-N,N-dimethylaniline $\left(C_{7}\right)$ :

IR $\left(\mathrm{KBr}, \mathrm{cm}^{-1}\right): 3049$ (=C-H str, aromatic), $1649(\mathrm{C}=\mathrm{N}$ str, pyrimidine) 1604 and $1427(\mathrm{C}=\mathrm{C}$ str, aromatic), 754 (C-Cl str), 617 (C-S-C str); ${ }^{1} \mathbf{H}$ NMR (400 Hz, DMSO$\left.\mathrm{d}_{6}\right) \delta \mathrm{ppm}: 2.28\left(\mathrm{~s}, 6 \mathrm{H}, 2 \mathrm{CH}_{3}\right), 3.06\left(\mathrm{~s}, 6 \mathrm{H}, \mathrm{Ar}-\mathrm{N}\left(\mathrm{CH}_{3}\right)_{2}\right)$, 3.99 (s, 2H, $\mathrm{CH}_{2}$ ), 7.22 (s, 1H, CH), 7.26-8.00 (m, 8H, Ar-H); EI-MS: 398; Ana. Calcd. for $\mathrm{C}_{21} \mathrm{H}_{23} \mathrm{ClN}_{4} \mathrm{~S}$ : C, 63.22; $\mathrm{H}$, 5.81; N, 14.04. Found C, 63.19; H, 5.79; N, 14.01\%.

4-(6-(4-chlorophenyl)-2-(((diethylamino)methyl)thio)pyrimidin-4-yl)- $N$, $N$-dimethylaniline $\left(C_{8}\right)$ :

IR $\left(\mathrm{KBr}, \mathrm{cm}^{-1}\right)$ : 3047 (=C-H str, aromatic), 1647 (C=N str, pyrimidine) 1605 and $1428(\mathrm{C}=\mathrm{C}$ str, aromatic $), 757$ (C-Cl str), 621 (C-S-C str); ${ }^{1}$ H NMR (400 Hz, DMSO$\left.\mathrm{d}_{6}\right) \delta$ ppm: $1.03\left(\mathrm{t}, 6 \mathrm{H}, 2 \mathrm{CH}_{3}\right), 2.64\left(\mathrm{q}, 4 \mathrm{H}, 2 \mathrm{CH}_{2}\right), 3.04$ (s, $\left.6 \mathrm{H}, \mathrm{Ar}-\mathrm{N}\left(\mathrm{CH}_{3}\right)_{2}\right), 4.02$ (s, 2H, $\left.\mathrm{CH}_{2}\right), 7.20$ (s, 1H, CH), 7.26-8.00 (m, 8H, Ar-H); EI-MS: 426; Ana. Calcd. for $\mathrm{C}_{23} \mathrm{H}_{27} \mathrm{ClN} 4 \mathrm{~S}: \mathrm{C}, 64.69 ; \mathrm{H}, 6.37 ; \mathrm{N}, 13.12$. Found $\mathrm{C}$, 64.65; H, 6.32; N, 13.11\%.

4-(6-(4-chlorophenyl)-2-((morpholinomethyl)thio)pyrimidin-4-yl)-N,N-dimethylaniline $\left(C_{9}\right)$ :

IR $\left(\mathrm{KBr}, \mathrm{cm}^{-1}\right): 3055$ (=C-H str, aromatic), $1645(\mathrm{C}=\mathrm{N}$ str, pyrimidine $) 1601$ and $1430(\mathrm{C}=\mathrm{C}$ str, aromatic $), 761$ (C-Cl str), 619 (C-S-C str); ${ }^{1} \mathbf{H}$ NMR (400 Hz, DMSO$\left.\mathrm{d}_{6}\right) \delta$ ppm: $2.96\left(\mathrm{t}, 4 \mathrm{H}, 2 \mathrm{CH}_{2}\right), 3.06\left(\mathrm{~s}, 6 \mathrm{H}, \mathrm{Ar}-\mathrm{N}\left(\mathrm{CH}_{3}\right)_{2}\right)$, $3.66\left(\mathrm{t}, 4 \mathrm{H}, 2 \mathrm{CH}_{2}\right), 4.01\left(\mathrm{~s}, 2 \mathrm{H}, \mathrm{CH}_{2}\right), 7.21(\mathrm{~s}, 1 \mathrm{H}, \mathrm{CH})$, 7.25-8.00 (m, 8H, Ar-H); EI-MS: 440; Ana. Calcd. for $\mathrm{C}_{23} \mathrm{H}_{25} \mathrm{ClN}_{4}$ OS: C, 62.64; H, 5.71; N, 12.70. Found C, 62.61; H, 5.69; N, 12.68\%.

4-(6-(4-chlorophenyl)-2-(((dimethylamino)methyl)thio)pyrimidin-4-yl)phenol $\left(C_{10}\right)$ :

IR $\left(\mathrm{KBr}, \mathrm{cm}^{-1}\right)$ : 3320 (O-H str), 3055 (=C-H str, aro- 
matic), $1645(\mathrm{C}=\mathrm{N}$ str, pyrimidine) 1601 and $1430(\mathrm{C}=\mathrm{C}$ str, aromatic), 755 (C-Cl str), 619 (C-S-C str); ${ }^{1} \mathrm{H}$ NMR $\left(400 \mathrm{~Hz}, \mathrm{DMSO}-\mathrm{d}_{6}\right) \delta \mathrm{ppm}: 2.27\left(\mathrm{~s}, 6 \mathrm{H}, 2 \mathrm{CH}_{3}\right), 3.98(\mathrm{~s}$, $\left.2 \mathrm{H}, \mathrm{CH}_{2}\right), 5.35$ (s, $\left.1 \mathrm{H}, \mathrm{Ar}-\mathrm{OH}\right), 7.23(\mathrm{~s}, 1 \mathrm{H}, \mathrm{CH}), 7.27-8.02$ (m, 8H, Ar-H); EI-MS: 371; Ana. Calcd. for $\mathrm{C}_{19} \mathrm{H}_{18} \mathrm{ClN}_{3} \mathrm{OS}$ : C, 61.36; H, 4.88; N, 11.30. Found C, 61.32; H, 4.82; N, $11.29 \%$.

\section{4-(6-(4-chlorophenyl)-2-(((diethylamino)methyl)thio)pyri- midin-4-yl)phenol $\left(C_{11}\right)$ : \\ IR $\left(\mathrm{KBr}, \mathrm{cm}^{-1}\right): 3325(\mathrm{O}-\mathrm{H}$ str), 3055 (=C-H str, aro-} matic), $1645(\mathrm{C}=\mathrm{N}$ str, pyrimidine) 1601 and $1430(\mathrm{C}=\mathrm{C}$ str, aromatic), 765 (C-Cl str), 619 (C-S-C str); ${ }^{1} \mathrm{H}$ NMR $\left(400 \mathrm{~Hz}, \mathrm{DMSO}-\mathrm{d}_{6}\right) \delta \mathrm{ppm}: 1.02\left(\mathrm{t}, 6 \mathrm{H}, 2 \mathrm{CH}_{3}\right), 2.66(\mathrm{q}$, $\left.4 \mathrm{H}, 2 \mathrm{CH}_{2}\right), 3.99\left(\mathrm{~s}, 2 \mathrm{H}, \mathrm{CH}_{2}\right), 5.33(\mathrm{~s}, 1 \mathrm{H}, \mathrm{Ar}-\mathrm{OH}), 7.20$ (s, 1H, CH), 7.26-8.01 (m, 8H, Ar-H); EI-MS: 399; Ana. Calcd. for $\mathrm{C}_{21} \mathrm{H}_{22} \mathrm{ClN}_{3} \mathrm{OS}$ : C, 63.36; $\mathrm{H}, 5.54 ; \mathrm{N}, 10.51$. Found C, 63.32; H, 5.52; N, 10.49\%.

\section{4-(6-(4-chlorophenyl)-2-(morpholinomethyl)thio)pyrimi- din-4-yl)phenol $\left(C_{12}\right)$ : \\ IR $\left(\mathrm{KBr}, \mathrm{cm}^{-1}\right): 3329(\mathrm{O}-\mathrm{H}$ str), 3055 (=C-H str, aro-} matic), $1645(\mathrm{C}=\mathrm{N}$ str, pyrimidine) 1601 and $1430(\mathrm{C}=\mathrm{C}$ str, aromatic), 769 (C-Cl str), 619 (C-S-C str); ${ }^{1}$ H NMR (400 Hz, DMSO-d 6 ) $\delta$ ppm: $2.96\left(\mathrm{t}, 4 \mathrm{H}, 2 \mathrm{CH}_{2}\right), 3.69(\mathrm{t}$, $\left.4 \mathrm{H}, 2 \mathrm{CH}_{2}\right), 3.97\left(\mathrm{~s}, 2 \mathrm{H}, \mathrm{CH}_{2}\right), 5.31(\mathrm{~s}, 1 \mathrm{H}, \mathrm{Ar}-\mathrm{OH}), 7.21$ (s, 1H, CH), 7.27-8.01 (m, 8H, Ar-H); EI-MS: 413; Ana. Calcd. for $\mathrm{C}_{21} \mathrm{H}_{20} \mathrm{ClN}_{3} \mathrm{O}_{2} \mathrm{~S}$ : C, 60.94; H, 4.87; N, 10.15 . Found C, 60.91; H, 4.85; N, 10.12\%.

\section{1-((4-(4-chlorophenyl)-6-(3-nitrophenyl)pyrimidin-2-} $y$ l) thio)- $N, N$-dimethylmethanamine $\left(C_{13}\right)$ :

IR $\left(\mathrm{KBr}, \mathrm{cm}^{-1}\right): 3061$ (=C-H str, aromatic), $1650(\mathrm{C}=\mathrm{N}$ str, pyrimidine) 1606 and $1428(\mathrm{C}=\mathrm{C}$ str, aromatic), 1525 and $1350(\mathrm{~N}=\mathrm{O}$ str, aromatic), $760(\mathrm{C}-\mathrm{Cl}$ str), $619(\mathrm{C}-\mathrm{S}-$ $\mathrm{C}$ str); ${ }^{1} \mathbf{H}$ NMR (400 Hz, DMSO-d 6 ) $\delta$ ppm: 2.26 (s, 6H, $\left.2 \mathrm{CH}_{3}\right), 4.00\left(\mathrm{~s}, 2 \mathrm{H}, \mathrm{CH}_{2}\right), 7.25(\mathrm{~s}, 1 \mathrm{H}, \mathrm{CH}), 7.29-8.05(\mathrm{~m}$, $8 \mathrm{H}, \mathrm{Ar}-\mathrm{H})$; EI-MS: 400; Ana. Calcd. for $\mathrm{C}_{19} \mathrm{H}_{17} \mathrm{ClN}_{4}$ $\mathrm{O}_{2} \mathrm{~S}: \mathrm{C}, 56.93 ; \mathrm{H}, 4.27 ; \mathrm{N}, 13.98$. Found $\mathrm{C}, 56.92 ; \mathrm{H}, 4.25$; $\mathrm{N}, 13.95 \%$.

\section{N-((4-(4-chlorophenyl)-6-(3-nitrophenyl)pyrimidin-}

\section{2-yl)thio)methyl)- $\mathrm{N}$-ethylethanamine $\left(C_{14}\right)$ :}

IR $\left(\mathrm{KBr}, \mathrm{cm}^{-1}\right): 3052$ (=C-H str, aromatic), $1647(\mathrm{C}=\mathrm{N}$ str, pyrimidine) 1601 and $1429(\mathrm{C}=\mathrm{C}$ str, aromatic $), 1528$ and $1352(\mathrm{~N}=\mathrm{O}$ str, aromatic), $751(\mathrm{C}-\mathrm{Cl}$ str), $618(\mathrm{C}-\mathrm{S}-$ C str); ${ }^{1} \mathbf{H}$ NMR $\left(400 \mathrm{~Hz}\right.$, DMSO-d $\left.\mathrm{d}_{6}\right) \delta$ ppm: 1.05 (s, 6H, $\left.2 \mathrm{CH}_{3}\right), 2.64$ (q, $4 \mathrm{H}, 2 \mathrm{CH}_{2}$ ), 3.99 (s, $\left.2 \mathrm{H}, \mathrm{CH}_{2}\right), 7.23$ (s, $1 \mathrm{H}$, CH), 7.26-8.01 (m, 8H, Ar-H); EI-MS: 428; Ana. Calcd. for $\mathrm{C}_{21} \mathrm{H}_{21} \mathrm{ClN}_{4} \mathrm{O}_{2} \mathrm{~S}: \mathrm{C}, 58.80 ; \mathrm{H}, 4.93 ; \mathrm{N}, 13.06$. Found $\mathrm{C}$, $58.79 ; \mathrm{H}, 4.91 ; \mathrm{N}, 13.02 \%$.

\section{$\mathrm{N}$-(((4-(4-chlorophenyl)-6-(3-nitrophenyl)pyrimidin-}

\section{2-yl)thio)methyl)morpholine $\left(C_{15}\right)$ :}

IR $\left(\mathrm{KBr}, \mathrm{cm}^{-1}\right): 3058(=\mathrm{C}-\mathrm{H}$ str, aromatic), $1645(\mathrm{C}=\mathrm{N}$ str, pyrimidine) 1606 and $1429(\mathrm{C}=\mathrm{C}$ str, aromatic), 1530 and $1354(\mathrm{~N}=\mathrm{O}$ str, aromatic), $762(\mathrm{C}-\mathrm{Cl}$ str $), 616(\mathrm{C}-\mathrm{S}-$ C str); ${ }^{1}$ H NMR (400 Hz, DMSO-d 6 ) $\delta$ ppm: 2.95 (t, 4H, $\left.2 \mathrm{CH}_{2}\right), 3.67\left(\mathrm{t}, 4 \mathrm{H}, 2 \mathrm{CH}_{2}\right), 4.01\left(\mathrm{~s}, 2 \mathrm{H}, \mathrm{CH}_{2}\right), 7.23(\mathrm{~s}, 1 \mathrm{H}$, CH), 7.28-8.05 (m, 8H, Ar-H); EI-MS: 442; Ana. Calcd. for $\mathrm{C}_{21} \mathrm{H}_{19} \mathrm{ClN}_{4} \mathrm{O}_{3} \mathrm{~S}: \mathrm{C}, 56.95 ; \mathrm{H}, 4.32 ; \mathrm{N}, 12.65$. Found $\mathrm{C}$, $56.93 ; \mathrm{H}, 4.31 ; \mathrm{N}, 12.63 \%$.

\section{CONCLUSION}

The S-Mannich bases of 4,6-diaryl-3,4-dihydropyrimidin-2(1H)-thiones were synthesised by a facile method. All the synthesised compounds $\left(\mathbf{C}_{\mathbf{1}}-\mathbf{C}_{\mathbf{1 5}}\right)$ were evaluated for their anti-ulcer and antibacterial activities. Compounds $\mathbf{C}_{\mathbf{4}}, \mathbf{C}_{\mathbf{5}}, \mathbf{C}_{\mathbf{6}}, \mathbf{C}_{\mathbf{1 4}}$ and $\mathbf{C}_{\mathbf{1 5}}$ exhibited more potent anti-ulcer activity; compounds $\mathbf{C}_{6}, \mathbf{C}_{\mathbf{9}}, \mathbf{C}_{\mathbf{1 2}}$ and $\mathbf{C}_{\mathbf{1 5}}$ have more potent antibacterial activity.

Acknowledgments. The authors are thankful to UCPSc, Kakatiya University for facilities. One of the authors (K.Venkateshwarlu) is grateful to the management of Vaagdevi College of Pharmacy for their help and encouragement. And the publication cost of this paper was supported by the Korean Chemical Society.

\section{REFERENCES}

1. Sushil kumar, B. S.; Devanand, S. B. Acta Pharm. 2003, 53, 223.

2. Sham, M.; Sondhi, N. S.; Monika, J.; Ashok Kumar, B. Med. Chem. 2005, 13, 6158.

3. Dravyakar, B. R.; Kawade, D. P.; Bhusari, K. P. Ind. J. Heterocycl. Chem. 2007, 16, 301.

4. Nagaraj, A.; Sanjeev Reddy, C. Iran. Chem. Soc. 2008, 5, 262.

5. Rathod, I. S.; Baheta, K. G. Indian J. Pharm. Sci. 2005, 67, 593.

6. Brun, O.; Ballabeni, V.; Barocellin, E. Biorg. Med. Chem. 2004, 12, 553.

7. Sinythises, J. R. Psychoneuroendocrinology. 1979, 4, 177.

8. Lavilla, R. J. Chem. Soc. 2002, 1, 1141.

9. Patil, P. A.; Bhole, R. P.; Chikhale, R. V.; Bhusari, K. P. Inter. J. Chem Tech Res. 2009, 1, 373.

10. Vogle, A. I.; Tachell, A. R.; Furnis, B. S.; Hannaford, A. J.; Smith, P. W. G. Vogel's Book of Practical Organic Chem- 
istry, 5th ed.; Prentice Hall: 1996, p 1034.

11. Amit, R. T.; Dipti, K. D; Naresh, R. R.; Viresh, H. S. ARKIVOC 2008, 11, 131.

12. Shah, T. B.; Gupte, A.; Patel, M. R.; Chaudhari, V. S.; Patel, H.; Patel, V. C. Ind. J. Chem. 2009, 48B, 88.

13. Barrett, W. E.; Rutledge, R.; Plummer, A. J.; Yonkman, F. F. J. Pharmacol. Exp. Ther. 1953, 108, 305.

14. Hemmati, M.; Razvani, A.; Diahanguini, B. Pharmacology
1973, 9, 374.

15. Shay, H.; Komarow, S. A.; Fels, S. S.; Meranze, D.; Gruenstein, M.; Siplet, H. Gastroenterol. 1973, 5, 43.

16. Gerhard Vogel, H. Drug Discovery and Evaluation, 2nd ed.; Springers: 2002.

17. Fung,T. J. C.; Minassian, B. Antimicrob Agents Chemother 2000, 44, 3351. 